\title{
A Numerical Study on Aerodynamic Noise Sources of High-Speed Train
}

\author{
Yan Yang* and Guowei Yang \\ LHD, Institute of Mechanics, Chinese Academy of Sciences, Beijing, China \\ yangy@ustc.edu
}

\begin{abstract}
Firstly, in order to investigate the noise generation mechanisms of the pantograph, the key source in aerodynamic noise generation, the basic models of a circular cylinder and a series of rectangular cylinders were numerical studied respectively and compared in detail. Secondly, the aerodynamic noise sources of a high-speed train were visualized, through the numerical simulation of a real threecoach high-speed train. Finally, the far-field noise levels are given in $350 \mathrm{~km} / \mathrm{h}$ and $400 \mathrm{~km} / \mathrm{h}$. Based on the results, flow and noise source generation mechanisms on the high-speed train were revealed.
\end{abstract}

Keywords: High-speed train, aerodynamic noise, noise source generation, CFD, CAA.

\section{Introduction}

In the last several decades, high-speed railway becomes to a modern transportation tool of more and more importance in the world. With the increase of the travelling speed of high-speed trains, the problem on aerodynamic noise appears more severe. As has been well-known, the aerodynamic noise levels increases at around $60 \log _{10} V$ (with $V$ the speed), on the other hand rolling noise levels increase at a rate of $30 \log _{10} V$, consequently the aerodynamic noise becomes dominant above a certain speed, named as the transition speed (Thompson, 2009). Therefore, when running above the certain speed (around $300 \mathrm{~km} / \mathrm{h}$ ), not only exterior noise emitted to the environment, but also interior noise transmitted to the passengers, mostly come from aerodynamic noise sources.

Aerodynamic noise sources in high-speed trains consist of various types (Talotte, 2000). Many engineering experiences and experimental studies indicate that pantograph is one of the most significant aerodynamic noise sources (King III, 1996; Talotte et al., 2003). The most promising noise reduction solution is to control the sound sources, through the shape optimization according to its aerodynamic and aeroacoustic characteristics. Though some studies aiming at aerodynamic noise from pantograph has been done, the basic flow mechanisms and sound source mechanisms still need deeper understanding, and the noise sources of real trains have not been simulated well by numerical model.

\footnotetext{
* Corresponding author.
} 
This paper set up two basic models from the structural element of pantograph, a circular cylinder and a series of rectangular cylinders to investigate the basic mechanisms of flow and noise sources generation, via CFD (Computational Fluid Dynamics) and CAA (Computational AeroAcoustics) methods. Based on the results, some flow and noise control concepts on the noise mitigation are proposed. With high performance computer simulation, real scale three-coach high-speed trains were simulated; noise sources were visualized via varieties of methods. The far-field noise levels were given.

\section{Physical Models}

Aerodynamic noise of the high-speed train is generated by the turbulent flow around the vehicle structures. Although the high-speed train surfaces have been smoothed much more than that of conventional trains, there are still many structural elements which inevitably interact with the incoming flow and constitute the aeroacoustic sources. The pantograph, roof structures, electronic insulators of the train are of one category of aeroacoustic sources, characterized by turbulent detachment and vortex shedding. The bogies are the most complex components of the train, which constructed another main aeroacoustic sources, characterized by strong turbulence and complex flow fields. Above structures are noted as the most important aerodynamic noise sources in literatures (King III, 1996; Talotte, 2000; Talotte et al., 2003).

Figure 1 shows the geometry of a typical pantograph, comprised of several structures of cylinders, of which some are circular and some are rectangular. The numerical simulations of the whole model of three-coach train suggest that in the pantograph region exist the most strong aeroacoustic sources. In order to investigate the sound generation mechanisms, two kinds of cylinder model should be set up. The first one is flow around a circular cylinder; the other is a series of rectangular cylinders, with the ratio of depth to height $(D / H)$ equal to $0.6,1,2.0$, 2.5 and 3.0. The models are chosen according to the nondimensional parameter $R e$ (Reynolds number), for comparing with the former computational study (Kravchenko and Moin, 2000; Rokugou et al., 2008). The grids of each model are presented in Figure 2.

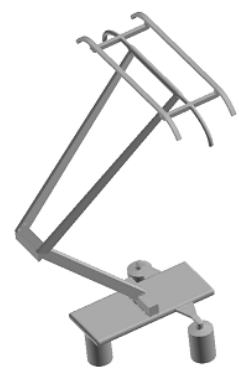

Fig. 1 Geometry of a pantograph 
For the real train model, because of the geometry complexity, the computational grid has to be unstructured, generally comprise prism, pyramid and tetrahedral elements.

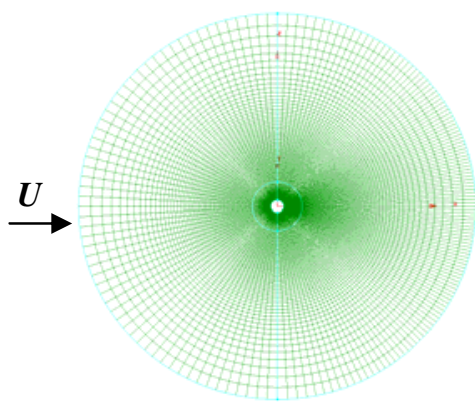

(a) Circular cylinder (global)

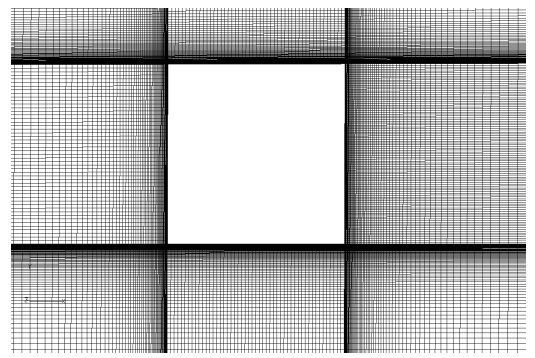

(b) Rectangular cylinder (local)

Fig. 2 Computational grids of the cylinder models

\section{Method of Solution}

Direct numerical simulation of noise source and its propagation simultaneously is impossible at present, especially in problems of complex body such as train. The state-of-the-art strategy of computational aeroacoustics is hybrid method that is implementing CFD in noise source region simulation and Lighthill's acoustic analogy in far-field noise computation.

\subsection{Governing Equations}

The governing equations in the source region are the spatially filtered NavierStokes equations of incompressible unsteady flow, with LES (Large Eddy Simulation) technique:

$$
\begin{gathered}
\frac{\partial}{\partial x_{i}} \overline{u_{i}}=0 \\
\frac{\partial \overline{u_{i}}}{\partial t}+\frac{\partial \overline{u_{i} u_{j}}}{\partial x_{j}}=-\frac{1}{\rho} \frac{\partial \bar{p}}{\partial x_{i}}+\frac{\partial}{\partial x_{j}}\left\{v\left(\frac{\partial \overline{u_{i}}}{\partial x_{j}}+\frac{\partial \overline{u_{j}}}{\partial x_{i}}\right)+\tau_{i j}\right\} .
\end{gathered}
$$

where $\bar{u}_{i}$ is the grid-scale velocity, $\bar{p}$ is the grid-scale static pressure, $\rho$ and $v$ are respectively the density and the kinematic viscosity of the air. The sub-grid scale eddies that not resolved by the grid are modelled after the Dynamic Smagorinsky Model (DSM) (Germano et al., 1991) modified by Lilly (Lilly, 1992). The details of the turbulence closure model formulae can be found in (Lilly, 1992). 
For far-field noise computation, the Lighthill's acoustic analogy (Lighthill, 1952) is implemented. According to (Curle, 1955), the sound pressure $p_{\mathrm{a}}$ is computed via

$$
p_{\mathrm{a}}=\frac{1}{4 \pi c_{0}} \frac{x_{i}}{r^{2}} \frac{\partial}{\partial t} \int_{S} \frac{n_{i} p\left(\boldsymbol{y}, t-r / c_{0}\right)}{r} d S .
$$

\subsection{Numerical Methods}

The numerical discretization method is Taylor-Galerkin FEM (Finite Element Method) with a streamline upwind scheme which has second accuracy both in space and time. The spatial discretization is performed by hexahedral mesh and the coordinate system is the 3-D Cartesian. The pressure algorithm is based on the Fractional step method. The linear equations (from the momentum equations and pressure Poisson equation) are solved by Bi-CGSTAB and Jacobi iteration methods.

\section{Results and Discussions}

\subsection{Circular Cylinder and Rectangular Cylinders}

This sub-section is also validations of the numerical methods, because all of the cases were chosen according to the references and the results were compared with them in detail.

The middle-section two-dimentional grids of the models are shown in Figure 2, where $\mathrm{x}$ is the direction of incoming flow, $\mathrm{z}$ is cross-flow direction, and $\mathrm{y}$ is the span direction. In the circular cylinder model the Reynolds number is $R e_{D}=3900$, where $D$ is the diameter; in the rectangular cylinder model, the Reynolds number is $R e_{H}=10000$, where $H$ is the height of rectangular cylinder. The outer inflow boundary condition is set as a constant inlet velocity; the outflow boundary is set as outlet; the span ends of the cylinder implements a cyclic condition. The span computation domain is chosen as $\pi D$ in the circular cylinder case, and $8 H$ in the rectangular cases.

The fluid force coefficients are defined as, lift coefficient $C_{L}=-2 F_{\mathrm{z}} / \rho U^{2} S_{\text {ref }}$, drag coefficient $C_{D}=-2 F_{\mathrm{x}} / \rho U^{2} S_{\text {ref }}$, where $S_{\text {ref }}$ is the frontal area of the body; the pressure coefficient is defined as $C_{P}=2\left(p-p_{\infty}\right) / \rho U^{2}$ and the base-pressure coefficient is defined as the pressure coefficient of the point of trailing edge.

Figure 3 gives the time history of the fluid dynamic parameters. And Table 1 presents the parameters, comparing with the reference. Figure 4 shows the velocity profile, compared with former numerical and experimental works. The results show good agreements with former works. Figure 5 shows the vortex structure in the wake. The vortex shedding phenomenon (Kármán vortex street) is presented clearly. According to Lighthill's acoustic equation, the periodic vortex shedding 
and the periodic oscillation of fluid forces, pressure and velocity fluctuation produce the dipole sound. Because vortex shedding is the main large scale fluctuation, the sound is characterized by the aeolian tone, the peak frequency of which should be coincident with the vortex shedding frequency.

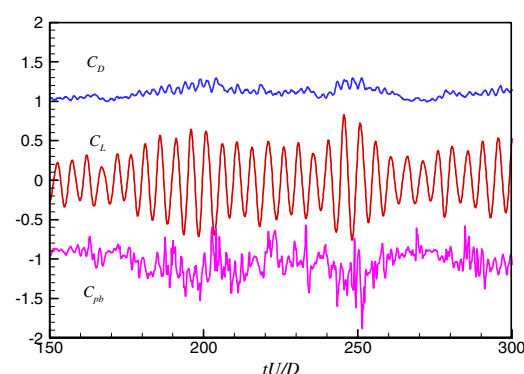

Fig. 3 Lift, drag and base-pressure coefficients of the flow with nondimensional time

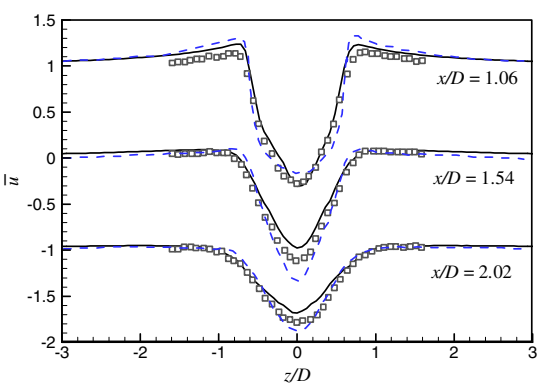

Fig. 4 Average stream direction velocity profile $\bar{u}$ with $\mathrm{z}$

Table 1 Flow parameters results (KM00 and Exp. from (Kravchenko and Moin, 2000))

\begin{tabular}{|c|c|c|c|c|c|c|c|c|c|}
\hline & St & $C_{D \text {,avg }}$ & $C_{D}, \mathrm{rms}$ & $C_{L}, \mathrm{rms}$ & $-C_{\mathrm{pb}, \text { avg }}$ & $C_{\mathrm{pb}, \mathrm{rms}}$ & $\begin{array}{c}\text { Detach } \\
\text { angle } \\
\theta_{\text {sep }}\end{array}$ & $\begin{array}{l}\text { Length } \\
\text { of } \\
\text { recurring } \\
L_{\mathrm{rec}} / \mathrm{D}\end{array}$ & $\begin{array}{l}\text { Stream } \\
\text { vel. } \\
\text { min. } \\
U_{\min }\end{array}$ \\
\hline Present & 0.204 & 1.13 & 0.068 & 0.356 & 1.06 & 0.19 & $85^{\circ}$ & - & -0.33 \\
\hline KM 00 & 0.210 & 1.04 & - & - & 0.94 & - & $88^{\circ}$ & 1.35 & -0.37 \\
\hline \multirow[t]{2}{*}{ Exp. } & 0.215 & 0.99 & - & - & 0.88 & - & $86^{\circ}$ & 1.4 & -0.24 \\
\hline & \pm 0.005 & \pm 0.05 & & & \pm 0.05 & & \pm 2 & \pm 0.1 & \pm 0.1 \\
\hline
\end{tabular}

Specify the kinematic viscosity $v$ equal to $1.5 \times 10^{-5} \mathrm{~m}^{2} / \mathrm{s}, U$ equal to $15 \mathrm{~m} / \mathrm{s}$ and $D$ equal to $3.9 \mathrm{~mm}$, the sound pressure at a far-field observer can be computed from equation (2). The result of the spectrum is shown in Figure 6. The peak frequency is $786 \mathrm{~Hz}$, corresponding sound pressure level is $37 \mathrm{~dB}$, which determines the total noise level, based on the acoustics theory. The frequency is coincident with the Strouhal number $\left(S t=f_{L} D / U\right)$ which computed from the $C_{L^{-}}$ time curve, that is to say, the dipole sound source induced by lift fluctuation is the predominant sound source in the flow over the circular cylinder. 

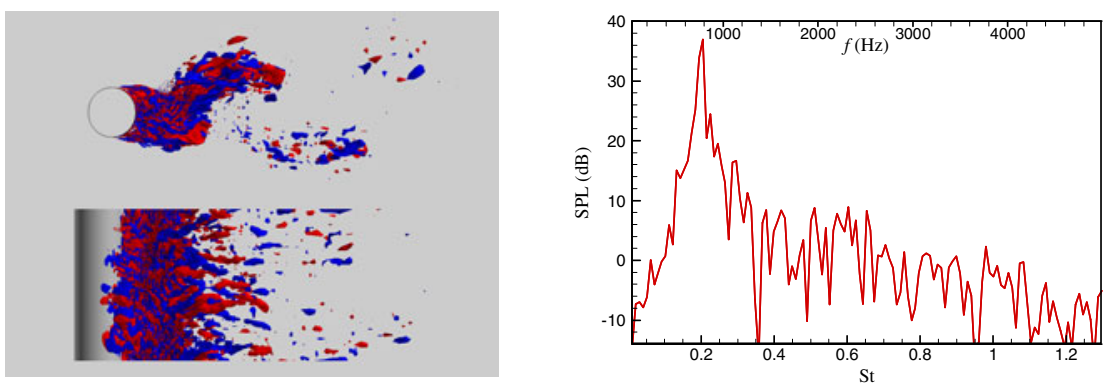

Fig. 5 Iso-surfaces of stream vorticity Fig. 6 Far-field noise spectrum observation at $\Omega_{\mathrm{x}}($ red denotes 8 , blue -8 ) $(0,0.5 \pi \mathrm{D}, 185 \mathrm{D})$

Figure 7 give the result of fluid dynamic parameters of the square cylinder $(D / H=1)$. The depth-height ratio $(D / H)$ effects are investigated by varying the value of $D$ with the fixed $H$. Figure 8 shows the results of Strouhal number varying with $\mathrm{D}$. The results agree well with the former works (Rokugou et al., 2008). The flow and fluid dynamic parameters exhibit a sudden change between $\mathrm{D} / \mathrm{H}$ equal to 2 and 3 . When the depth of the rectangular cylinder increase, the detachment of the boundary layer over the sides will be attenuated and reattachment will happen, therefore the lift fluctuation, pressure fluctuation and velocity fluctuation will be weaken.

As a result, the noise source strength should change accordingly, so as to farfield noise level, as Figure 9 shows. The spectrum of square cylinder has common characteristics with circular cylinder, where a peak frequency exists, corresponding to St number, and the total noise level is determined by the SPL at the frequency. However for the rectangular one with $D / H=3$, the peak frequency is not so noticeable and the SPL is far less that the square cylinder.

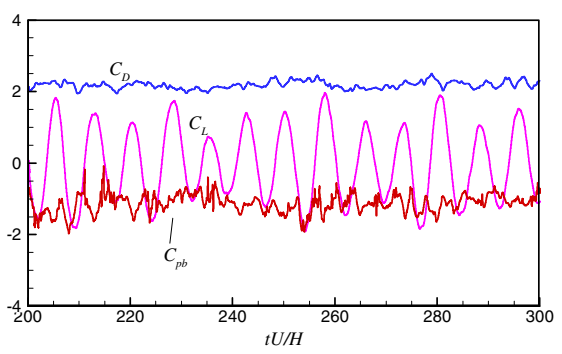

Fig. 7 Lift, drag and base-pressure coefficients of the flow with nondimensional time $(D / H=1)$

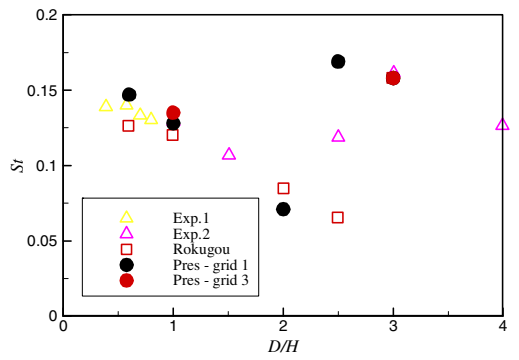

Fig. 8 St of lift coefficient versus depthheight ratio 

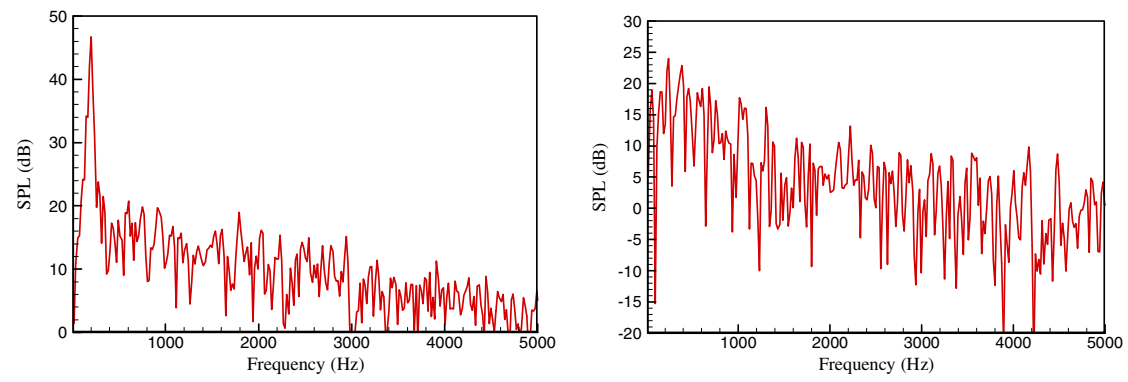

(a) Square cylinder $(D / H=1)$

(b) Rectangular cylinder with $D / H=3$

Fig. 9 Far-field (0, 0, 185D) sound pressure level spectrum of rectangular cylinder

\subsection{Noise Sources of High-Speed Train}

To investigate the sources of high-speed train, variety of methods exists. Here, three different quantities are implemented to identify the sources. The first quantity is the turbulent kinetic energy (TKE),

$$
k=\frac{1}{2}\left(\bar{u}^{2}+{\overline{v^{\prime}}}^{2}+{\overline{w^{\prime}}}^{2}\right),
$$

which can be computed in RANS with some turbulence models, such as $k-\varepsilon$ model. The second quantity is the right hand term of Howe's vortex sound function (Howe, 2003)

$$
\left(\frac{1}{c_{0}^{2}} \frac{\partial^{2}}{\partial t^{2}}-\nabla^{2}\right) B=\nabla \cdot(\boldsymbol{\omega} \times \boldsymbol{u}), \quad B=\int \frac{d p}{\rho}+\frac{1}{2} u^{2} .
$$

called vortex-sound source here, where $B$ is called as the total enthalpy. The third is the overall sound pressure levels, expressed as

$$
\text { OASPL }=20 \log _{10}\left(p^{\prime}{ }_{\text {rms }} / p_{\text {ref }}\right),
$$

where $p$ ' is the sound pressure, the quantity can be computed both in the field and on the body surface, $p_{\text {ref }}=2.0 \times 10^{-5}$. When the field is computed by LES, $p$ ' is defined as $p^{\prime}(t)=p(t)-p_{\text {avg }}$, where $p$ is the instantaneous flow pressure, and $p_{\text {avg }}$ is the time-averaged pressure. The definition is not strictly equal to the sound pressure, though it can be used to identify the sound when the numerical simulation is accurate relatively.

Figure 10 and Figure 11 shows the visualization of a test train, the former through equation (3), and the later through equation (5). Figure 12 shows the noise source visualization of another train with pantograph with a shield. These 
visualization results are similar qualitatively, which can indicate the localization of noise sources on the surface of high-speed train. Results suggest that the main noise sources are successively: the pantograph, bogies, roof structures (including the pantograph shield and insulators), inter-coach spacing, the nose and the tail. The results are mainly agreed with preceding measurements (King III, 1996; Talotte, 2000; Talotte et al., 2003).

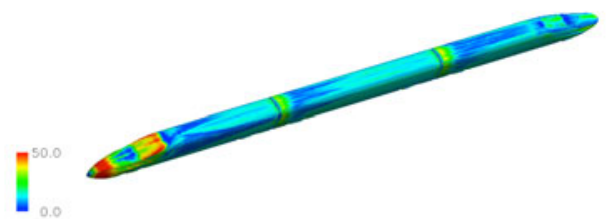

Fig. 10 Turbulent kinetic energy on the train surface

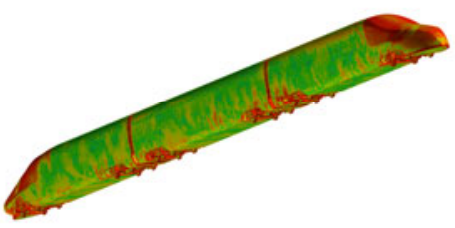

Fig. 11 OASPL on the train surface

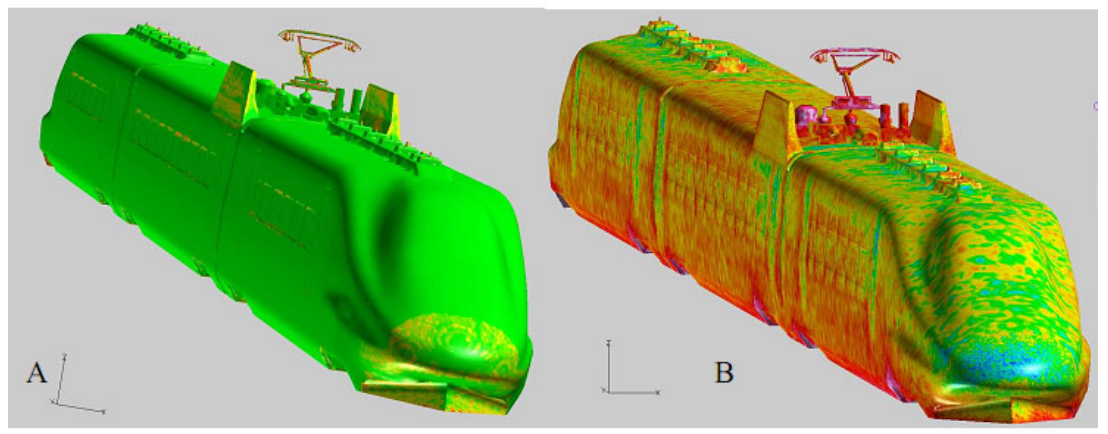

Fig. 12 Noise source visualization of a train, (A) vortex-sound source (B) OASPL

\subsection{Far-Field Noise of High-Speed Train}

Figure 13 gives the far-field noise of the former high-speed train model under two velocity levels, $350 \mathrm{~km} / \mathrm{h}$ and $400 \mathrm{~km} / \mathrm{h}$. The distribution of far-field noise level is characterized by the peak value at the head-coach. Figure 14 gives the A-weighted noise level spectrum at the observation, with coordinate $(0,25,3.5) \mathrm{m}$. The notable noise level bands exist between 100 500 Hz. 


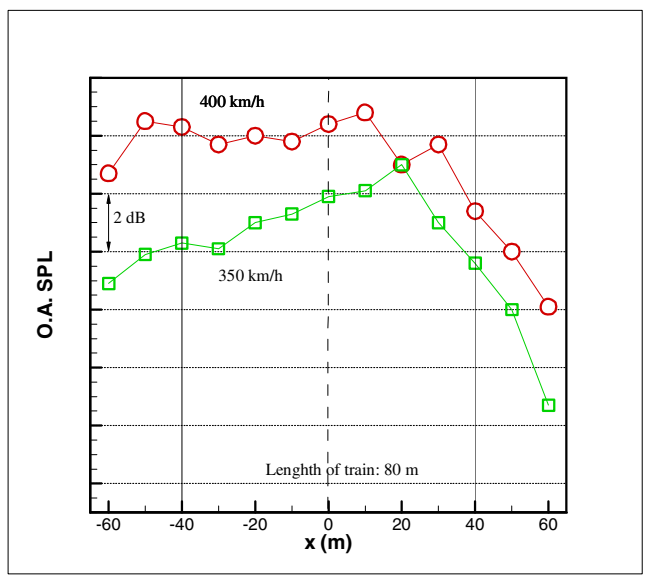

Fig. 13 Far-field noise level of the train at $25 \mathrm{~m}$ to the track and $3.5 \mathrm{~m}$ height.( $\mathrm{x}$ is the train head direction)

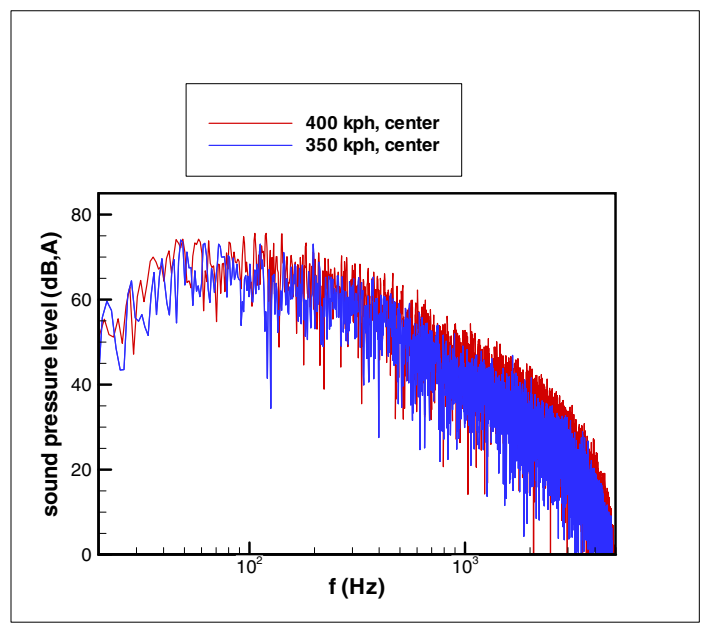

Fig. 14 Far-field A-weighted SPL at the centered observation point

\section{Conclusions}

The paper has investigated two basic aeroacoustic models, circular cylinder and rectangular cylinders, of pantograph, which is the most important aerodynamic noise source of high-speed train. The real train simulations haven been completed, and the noise sources were visualized. Base on the results, two folds of conclusions are given below 
1) Aeolian tone induced by cylinder in the wind is a universal sound source typically in the structural elements of high-speed train, its main sound source mechanism is dipole sound induced by the vortex shedding and detachment of the boundary layer; for cylinders, the section shape variety can cause notable changes in the unsteady flow, fluid dynamic parameters and sound source strength, therefore new flow and noise control method should be found by optimization on the section shape and span alteration.

2) Noise sources visualization via CFD helps identify the noise intensity of noise sources and design of train structural elements.

3) The notable noise level frequency bands of the far-field noise exist between $100 \sim 500 \mathrm{~Hz}$.

Acknowledgment. The authors gratefully acknowledge the financial support of 973 Program (2011CB711100), National Key Technology R\&D Program (2009BAG12A03) and Project of Knowledge Innovation Engineering (KJCX2-EW-L02-1) of CAS.

\section{References}

Curle, N.: The influence of solid boundaries upon aerodynamic sound. Proceedings of the Royal Society of London. Series A, Mathematical and Physical Sciences 231(1187), 505-514 (1955)

Germano, M., Piomelli, U., Moin, P., Cabot, W.H.: A dynamic subgrid-scale eddy viscosity model. Physics of Fluids A: Fluid Dynamics 3(7), 1760-1765 (1991)

Howe, M.S.: Theory of vortex sound. Cambridge University Press, New York (2003)

King III, W.F.: précis of developments in the aeroacoustics of fast trains. Journal of Sound and Vibration 193(1), 349-358 (1996)

Kravchenko, A.G., Moin, P.: Numerical studies of flow over a circular cylinder at Re= 3900. Physics of Fluids 12, 403 (2000)

Lighthill, M.J.: On sound generated aerodynamically. I. General theory. Proceedings of the Royal Society of London. Series A, Mathematical and Physical Sciences 211(1107), 564-587 (1952)

Lilly, D.K.: A proposed modification of the Germano subgrid-scale closure method. Physics of Fluids A: Fluid Dynamics 4, 633 (1992)

Rokugou, A., Kiwata, T., Okajima, A., Kimura, S., Yamamoto, H.: Numerical analysis of aerodynamic sound radiated from rectangular cylinder. Journal of Wind Engineering and Industrial Aerodynamics 96(10-11), 2203-2216 (2008)

Talotte, C.: Aerodynamic noise: A critical survey. Journal of Sound and Vibration 231(3), 549-562 (2000)

Talotte, C., Gautier, P.E., Thompson, D.J., Hanson, C.: Identification, modelling and reduction potential of railway noise sources: A critical survey. Journal of Sound and Vibration 267(3), 447-468 (2003)

Thompson, D.: Railway noise and vibration: Mechanisms, modelling and means of control. Elsevier, Oxford (2009) 